\title{
TEMPORAL EVOLUTION OF INTRACRANIAL HEMORRHAGE OOCIATED WITH VITAMIN K ANTAGONISTS IN SF TAO
FROM TAC RESGISTRY 1 TO TAC REGISTRY 2 Thesiny
}

C. Aguirre1, G. Zapata-Wainberg ${ }^{1}$, S. Quintas ${ }^{1}$, J. Masjuán Vallejo², L. Benavente Fernández ${ }^{3}$, J. Egido4 ${ }^{4}$ MM. Freijó5, P. Cardona ${ }^{6}$, J. Gallego Culleré ${ }^{7}$, MM Castellanos Rodríguez ${ }^{8}$, A. García Pastor ${ }^{9}$, JC Gómez Sánchez ${ }^{10}$ J Maciñeiras ${ }^{11}$, J. Vivancos ${ }^{1}$.

On behalf of TAC-Registry 1 and 2 Investigators. Spain

1 Stroke Unit, Neurology Department. Hospital Universitario de La Princesa, Princesa Health Research Institute Madrid. 2 Stroke Unit, Neurology Department. Hospital Universitario Ramón y Cajal, Madrid.3 Stroke Unit, Neurology Department. Hospital Universitario Central de Asturias, Oviedo. 4 Stroke Unit, Neurology Department .Hospital Universitario Clínico San Carlos, Madrid. 5 Stroke Unit, Neurology Department. Hospital de Basurto, Bilbao. 6 Stroke Unit, Neurology Department. Hospital Universitario de Bellvitge 7 Stroke Unit, Neurology Department. Complejo hospitalario de Navarra. 8 Stroke Unit, Neurology Department. Complejo hospitalario Universitario de A Coruña. 9 Stroke Unit, Neurology Department. Hospital General Universitario Gregorio Marañón. 10 Stroke Unit, Neurology Department. Hospital Universitario de Salamanca. 11 Stroke Unit, Neurology Department. Complejo hospitalario de Vigo.

\section{BACKGROUND:}

To assess possible changes in epidemiology, characteristics and outcome of intracranial hemorrhage associated with vitamin $\mathrm{K}$ antagonists (ICH-VKA).

\section{RESULTS:}

\begin{tabular}{|l|l|l|} 
& \multicolumn{1}{c|}{ TR 1 } & \multicolumn{1}{c|}{ TR 2 } \\
\hline Number of hospitals & 21 & 29 \\
\hline Population (inhabitants) & 8.155 .628 & 11.864 .971 \\
\hline N & 235 & 331 \\
\hline Annual incidence ICH-VKA & $2,88 / 100.000$ & $2,79 / 100.000$ \\
\hline \% NRL admitted patients & $1,14 \%$ & 1,22 \\
\hline \% strokes & $1,94 \%$ & $2,04 \%$ \\
\hline \% ICH & $13,62 \%$ & $16,42 \%$ \\
\hline
\end{tabular}

Table 1. Demographic characteristics

\begin{tabular}{|l|l|l|l|} 
& \multicolumn{1}{|c|}{ TR 1 } & \multicolumn{1}{c|}{ TR 2} & \multicolumn{1}{c}{$P$} \\
\hline Average age (SD) & $78,17(9,39)$ & $77,69(8,43)$ & 0.52 \\
\hline Sex female (n) & $46 \%(108)$ & $39 \%(129)$ & 0.097 \\
\hline HTA (n) & $79,6 \%(187)$ & $80,1 \%(265)$ & 0.887 \\
\hline DM (n) & $27,2 \%(64)$ & $34,1 \%(113)$ & 0.81 \\
\hline AF $(n)$ & $80 \%(188)$ & $79,5 \%(263)$ & 0.874 \\
\hline
\end{tabular}

Table 2. Patients characteristics

\section{METHODS:}

We evaluate patients with ICH-VKA included in TAC-

Registry1(TR1) and TAC-Registry2(TR2) studies:

country-wide, retrospective, observational multicentric studies that included all adult patients with ICH associated with anticoagulation admitted to Neurology Departments. TR1: September 2012August 2013, TR 2: year 2015.

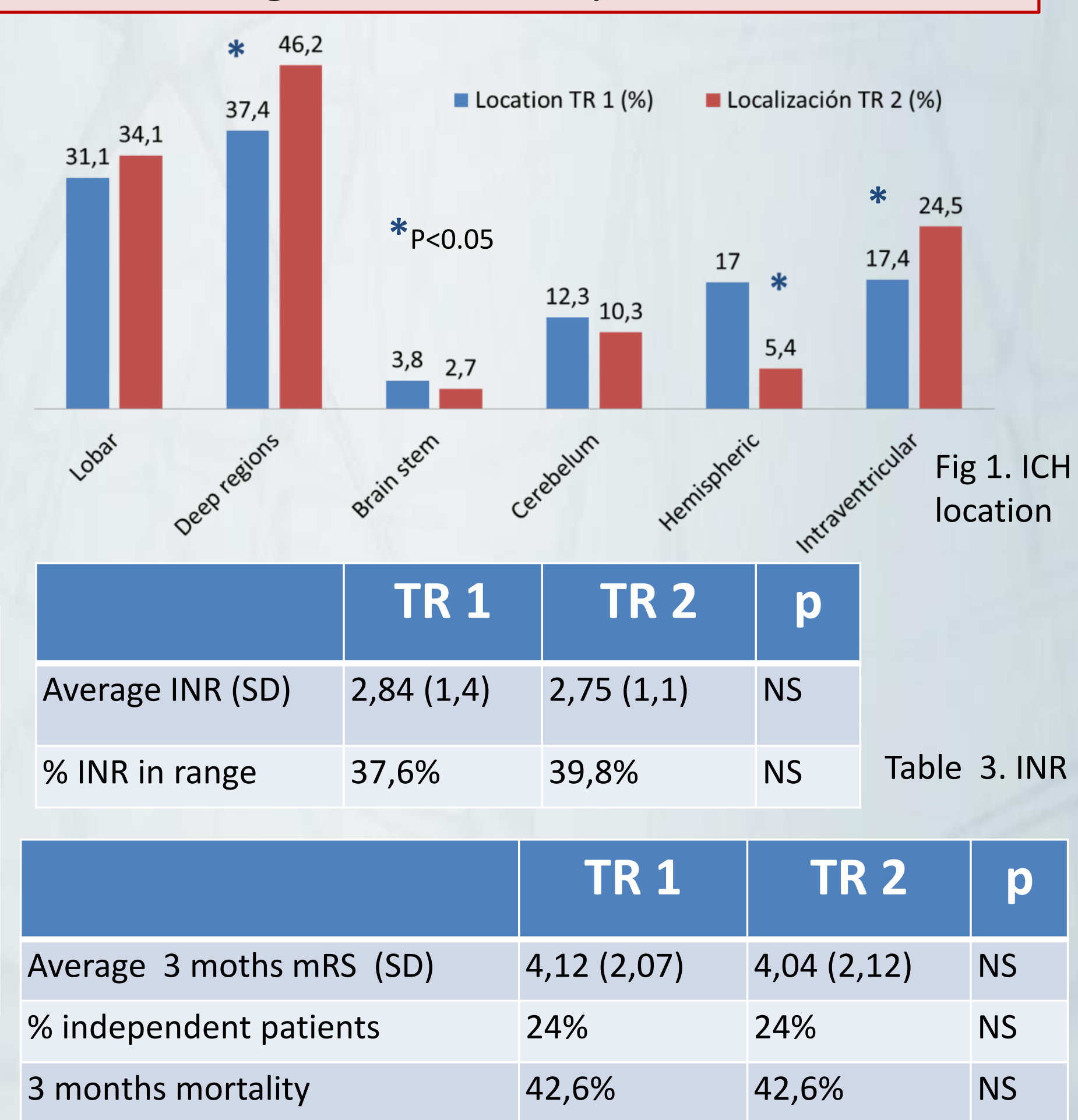

Table 4. Three months outcome

\section{CONCLUSIONS:}

ICH-VKA incidence remains stable in recent years. Nevertheless, its relative frequency among ICHs has increased. Its poor outcome and association with variable INR ranges remains constant, representing a problem still unsolved.

Hospital Universitario de La Princesa

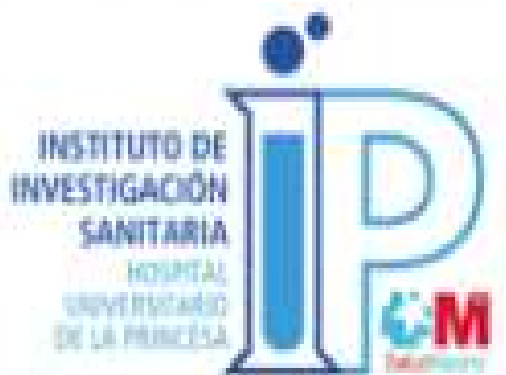

